\title{
Inseguridad Percibida en los Barrios de Santiago de Chile: La Importancia del Bienestar Subjetivo*
}

\author{
Maria Luisa Mendez Layera ${ }^{1}$ \\ Gabriel Otero² \\ Vania Perret ${ }^{3}$
}

1Professora associada ao Instituto de Estudios Urbanos y Territoriales. Pontificia Universidad Católica de Chile. Diretora do Centro de Estudios del Conflicto y la Cohesión Social, COES. Santiago, Chile.

E-mail: mmendezl@uc.cl, https:/ / orcid.org/0000-0002-3493-1511

${ }^{2}$ Escola de Sociologia da Universidade de Amsterdã. Amsterdã, Holanda.

E-mail: gabriel.otero@uva.nl, https:/ / orcid.org/0000-0001-9768-5699

${ }^{3}$ Socióloga pela Universidade Diego Portales. Santiago, Chile.

E-mail: vania.perret@mail.udp.cl, https:/ / orcid.org/0000-0002-2413-392X

\section{INTRODUCCIÓN}

I os sentimientos de inseguridad y miedo al crimen se han Linstalado como una preocupación sustantiva en los residentes y autoridades políticas de la ciudad de Santiago, pese a que la urbe más importante de Chile presenta indicadores relativamente moderados de criminalidad, en comparación con otras ciudades del mundo (Paydar et al., 2017). Se trata de una paradoja relevante, en cuanto la literatura ha constatado una variedad de consecuencias negativas asociadas con su extensión, sobre todo en ciudades marcadas por la desigualdad social y segregación espacial (Vilalta, 2011). La evidencia reportada desde diversas disciplinas muestra impactos negativos tanto en individuos como en comunidades, vinculados mayormente con trastornos en la salud física y mental de las personas (Ruijsbroek et al., 2015), pero también con una mayor propensión en la adopción de actitudes y comportamientos ofensivos, menor participación social y apoyo a políticas sociales, el aumento del gasto en dispositivos de seguridad y en servicios privados de vigilancia (Skogan, 1990). Esto ha llevado a algunos autores a señalar que el miedo al crimen

\footnotetext{
"Agradecemos el apoyo del Centro de Estudios de Conflicto y Cohesión Social - COES (CONICYT /FONDAP /15130009); CONICYT / FONDECYT proyecto 1191440; y CONICYT PFCHA/DOCTORADO BECAS CHILE/2017 - 72180515.
}

DADOS, Rio de Janeiro, vol.63(1):e20170036, 2020.

http://dx.doi.org/10.1590/001152582020200 
representa un problema mucho más grave que el crimen en sí mismo, puesto que, a diferencia de la delincuencia real, la inseguridad afecta a una mayor cantidad de personas, y sus consecuencias son aún más severas y extendidas en el tiempo (Hale, 1996; Warr, 1985). Autores tanto a nivel global (Judd, 1995; Lupton, 2000; Garland, 2008) como en Latinoamérica se han referido a la forma en que la sensación de inseguridad es producida por diversos factores, siendo un foco importante la producción social del miedo a través de estadísticas, medios de comunicación, agendas políticas, entre otras (Carrión y Núñez-Vega, 2006; Kessler, 2009; Dammert, 2012; Valdivia et al., 2012).

La investigación en torno a los sentimientos de inseguridad y miedo al crimen tiene sus orígenes en Estados Unidos en la década del sesenta, como respuesta a la preocupación por las crecientes tasas de criminalidad. En términos conceptuales, este fenómeno ha sido definido como un entramado de discursos, emociones, acciones y representaciones, respecto de amenazas asociadas a la ocurrencia de actos criminales y temores que traspasan lo delictual, donde se interrelacionan tanto aspectos objetivos como subjetivos (Ferraro, 1995; Hummelsheim et al., 2011; Kessler, 2009; LaGrange et al., 1992). Desde un principio, la literatura supuso que las creencias sobre la delincuencia podían explicarse a partir de la actividad criminal existente en los entornos geográficos inmediatos de las personas (Liska et al., 1982; Skogan, 1990). Sin embargo, una primera constatación, sorpresiva por lo demás, fue la inconsistente relación entre las percepciones subjetivas y los indicadores objetivos. En la práctica, los sentimientos de inseguridad de las personas parecen tener una autonomía relativa (Breetzke y Pearson, 2014; Brunton-Smith y Sturgis, 2011), tanto así que en un área determinada los residentes pueden sostener diferentes interpretaciones sobre la posibilidad de tener encuentros peligrosos (Gibson et al., 2002). Así pues, buena parte de los estudios ha demostrado que los temores de las personas en torno al delito, no estarían directamente relacionados con el riesgo objetivo de victimización, sino que más bien representarían un problema social más abstracto que contiene otros miedos e inseguridades de carácter económico, político y social de los ciudadanos (Amerio y Roccato, 2005; Dammert y Malone, 2006; Hale, 1996; Hummelsheim et al., 2010), además de percepciones sobre el medio ambiente, la estabilidad y el orden social (Garofalo, 1981; Gibson et al., 2002; LaGrange et al., 1992; Wyant, 2008).

En efecto, de acuerdo a lo subrayado por propuestas enfocadas en los procesos macrosociales, los sentimientos de inseguridad y miedo 
al crimen se han entendido dentro de un entramado analítico que subraya el vaciamiento de las estructuras económico-políticas, y el debilitamiento de la posición del Estado como institución garante del bienestar social. En este sentido, las percepciones en cuestión operarían como una esponja, absorbiendo todo tipo de ansiedades y temores vitales (Jackson, 2006). Esta interpretación más sociológica ha derivado de relatos que destacan la variedad de riesgos circunscritos a los grandes procesos modernizadores vividos en las últimas décadas, mayormente en las grandes ciudades, y en función de problemáticas como la creciente precariedad laboral, la disminución de los vínculos locales, la falta de solidaridad, así como el aumento de la importancia del mercado para asegurar las condiciones de vida de las personas (Bauman, 2000; Beck, 1999; Furedi, 2002).

Para el caso de Latinoamérica en especial, si bien desde la criminología se han intentado explicar los sentimientos de inseguridad y miedo al crimen mayormente en vinculación con el delito (Bergman y Flom, 2008; Caldeira, 2000; Dammert y Arias, 2007; Kessler, 2009), otros autores se han distinguido por relacionar este tipo de percepciones con las ansiedades propias de la modernización urbano-periférica, y específicamente con la herencia de las dictaduras militares en la región (Corradi et al., 1992; Koonings y Kruijt, 1999; Lechner, 1988; Reguillo, 2000). Estas perspectivas tienden a coincidir en el papel que también le cabe a distinciones clave del contexto latinoamericano como son las de clase social, y otros "estigmas operantes" como la raza y la edad (Lunecke, 2016:112). Complementariamente, la literatura ha supuesto que la expansión de los sentimientos de inseguridad y miedo al crimen estarían asociadas a campañas políticas y mediáticas, orientadas a promover el imaginario de que se trata de un problema que la política maneja con éxito, permitiendo así desviar la atención de los ciudadanos respecto de sus causas reales y otros problemas sociales, mayormente asociados con las dramáticas desigualdades socio-espaciales que exhibe la región a nivel comparado (Chevigny, 2003; Dammert, 2012; Dammert y Malone, 2006; Rotker y Goldman, 2002). Adicionalmente, hay estudios que enmarcan los sentimientos de inseguridad en un entramado mayor de cambio respecto de la propia subjetividad frente a procesos de segregación y fragmentación socio-espacial. A nivel de la vida barrial - al igual que en otras esferas de la vida social - se tendería a reintroducir mecanismos de diferenciación socio espacial en torno a códigos de conducta entre grupos al interior de las comunidades, y con ello, se reforzaría la desconfianza 
y el sentimiento de inseguridad frente a lo que aparece como peligroso (Lunecke, 2016; Dammert, 2012).

En cuanto a la evidencia disponible, las investigaciones han destacado una variedad de predictores específicos de los sentimientos de inseguridad y miedo al crimen, dependiendo de las disciplinas y enfoques que los estudian. Según gran parte de la literatura, estas percepciones son más probables en mujeres, población de mayor edad, minorías étnicas, no propietarios, solteros y grupos desventajados económicamente (Brunton-Smith y Sturgis, 2011; Hummelsheim et al., 2011; McNeeley y Yuan, 2017). También, cuando las personas han tenido experiencias de victimización previa, perciben que residen en barrios con un alto desorden físico y social, consideran que en su entorno no existe una adecuada provisión de servicios sociales, y existe desconfianza y poca sociabilidad con vecinos (Breetzke y Pearson, 2014; Donder et al., 2012; Hale, 1996; Hinkle, 2014; Zhao et al., 2015). Adicionalmente, se ha reportado la influencia negativa de contextos geográficos o barrios que, objetivamente, están caracterizados por una marcada segregación de bajos ingresos, mayores tasas de criminalidad, y carencia de la recursos institucionales para proveer de servicios básicos a la población residente (Breetzke y Pearson, 2014; Wilcox et al., 2003). Con todo, los hallazgos han sido consistentes respecto de que los factores subjetivos ejercen una mayor influencia sobre los sentimientos de inseguridad y miedo al crimen, que los indicadores objetivos (Kanan y Pruitt, 2002; Wyant, 2008).

En el contexto barrial, se han subrayado algunos mecanismos conceptuales para entender y explicar la extensión de los sentimientos de inseguridad y miedo al crimen, incorporando tanto factores sociales como del entorno construido (Paydar et al., 2017). En esta línea destacan especialmente las teorías de la vulnerabilidad física y social (Ferraro, 1995; Skogan, 1990), la victimización directa e indirecta (Hale, 1996; Killias y Clerici, 2000), y el enfoque de las "ventanas rotas" (broken windows) (Hinkle, 2014; Wilson y Kelling, 1982); aunque también se han subrayado algunos dispositivos de cohesión social como las redes, la confianza y el apoyo comunitario (Brunton-Smith y Sturgis, 2011), además de los tradicionales "efectos del barrio", mayormente asociados con la calidad de los entornos geográficos (van Ham et al., 2012), aunque también críticamente interpretados como formas de desprotección, desregulación y retirada de las autoridades gubernamentales de los sectores más desaventajados de las ciudades (López-Morales, 2016; Paydar et al., 2017; Slater, 2013). 
Siguiendo estos aportes, este artículo tiene como objetivo determinar los predictores objetivos y subjetivos de los sentimientos de inseguridad y miedo al crimen de los residentes de la ciudad de Santiago de Chile, contribuyendo así a complementar trabajos previos en el área (v.g. Dammert and Malone, 2003; Núñez et al., 2012, entre otros). Por un lado, proponemos un enfoque teórico integrador en torno al concepto de "bienestar", el cual permite rescatar tanto las dimensiones objetivas como subjetivas subrayadas en la literatura, así como los distintos contextos explorados: individuales y geográficos. Por otro lado, en términos metodológicos combinamos datos una encuesta representativa de 600 residentes, con datos georreferenciados del contexto barrial sobre los niveles de criminalidad, disponibilidad de recursos institucionales, y la concentración de pobreza/riqueza.

En síntesis, la investigación que presentamos pretende hacer contribuciones tanto en términos conceptuales como metodológicos. Primero, a raíz del poco consenso que representa la literatura en cuanto a las definiciones teóricas, integramos distintas aproximaciones que han buscado explicar los sentimientos de inseguridad y miedo al crimen, considerando su naturaleza multidimensional. Sostenemos que estos sentimientos no sólo deben ser entendidos como una respuesta al problema de la criminalidad, sino que, más bien representan diversas formas del bienestar de las personas y su entorno social, así como las texturas y percepciones que tienen respecto de las estructuras sociales y políticas que los producen y reproducen. Segundo, utilizamos una muestra representativa de toda una ciudad, a diferencia del énfasis habitual en la literatura centrado en entornos residenciales marginados o de bajos ingresos (Rollwagen, 2016). Tercero, se incluyen datos georreferenciados sobre los barrios, los que permiten resolver los problemas de endogeneidad que suelen caracterizar a las investigaciones que utilizan datos subjetivos agregados. Finalmente, nos enfocamos en un contexto distintivo y poco explorado, donde se combinan altos niveles de desigualdad social y segregación espacial (Link et al., 2015; LópezMorales, 2016; Méndez y Otero, 2018; Paydar et al., 2017).

\section{ORIENTACIONES TEÓRICAS E HIPÓTESIS DE INVESTIGACIÓN}

En esta investigación proponemos un enfoque que integra los aportes teóricos provenientes de distintas disciplinas. Siguiendo formulaciones anteriores (Hummelsheim et al., 2011), consideramos que las percepciones de inseguridad y miedo al crimen en el barrio pueden 
ser explicadas, más que por los niveles de criminalidad, por las diferentes condiciones económicas y sociopolíticas, donde también se incluyen percepciones subjetivas sobre el orden y la cohesión social. Para reunir estos distintos dispositivos, proponemos el concepto de "bienestar" como mecanismo integrador.

En general, las definiciones conceptuales y operacionales en torno al bienestar no sólo se han basado en las condiciones objetivas de vida como el ingreso, la estructura familiar, el acceso a vivienda digna, la educación de calidad y trabajo formal, sino también ha referido a un amplio abanico de dimensiones subjetivas, como la satisfacción, felicidad, confianza, participación cívica y las relaciones interpersonales (Delhey y Dragolov, 2016; Kroll y Delhey, 2013). De esa manera, incorpora no sólo actitudes y prácticas positivas, sino también experiencias afectivas negativas como la ansiedad y las preocupaciones vitales (Delhey et al., 2002). Mientras la dimensión objetiva del bienestar comprende aquellas cosas que se configuran con independencia de la conciencia individual, la dimensión subjetiva se ocupa de aspectos de la realidad que los individuos construyen y experimentan colectivamente (Kroll y Delhey, 2013). En este sentido, radica en evaluaciones sobre las condiciones que les rodean en sus comunidades y sociedades. Siguiendo esta lógica, se ha distinguido entre los contextos micro y macro, para referir a las condiciones de vida de los individuos y las condiciones sociales ancladas a ciertos entornos de referencia, respectivamente (Delhey et al., 2002). En suma, los estudios han subrayado el carácter multidimensional del concepto, donde se incluyen, conjuntamente, aspectos objetivos y subjetivos, cuestiones de riqueza material e inmaterial, y distintos niveles geográficos.

En los últimos años, la literatura emergente en torno al concepto de bienestar ha destacado aspectos específicos como la cohesión social y segregación geográfica, los cuales van más allá de las delimitaciones tradicionales orientadas a definir la satisfacción con distintos ámbitos de la vida (Berger-Schmitt, 2002; Delhey y Dragolov, 2016). Siguiendo estas consideraciones, nuestra propuesta teórica combina dos contextos: el individual y el barrial; y dos dimensiones: la objetiva y la subjetiva. En la práctica, identificamos cuatro mecanismos teóricos del bienestar con un anclaje especial en el entorno geográfico de influencia (ver Figura 1). En lo que sigue, abordamos cada uno de estos mecanismos teóricos y detallamos los factores que los componen. 
Figura 1

Mecanismos Teóricos del Bienestar en Asociación con el Miedo al Crimen o Sentimientos de Inseguridad

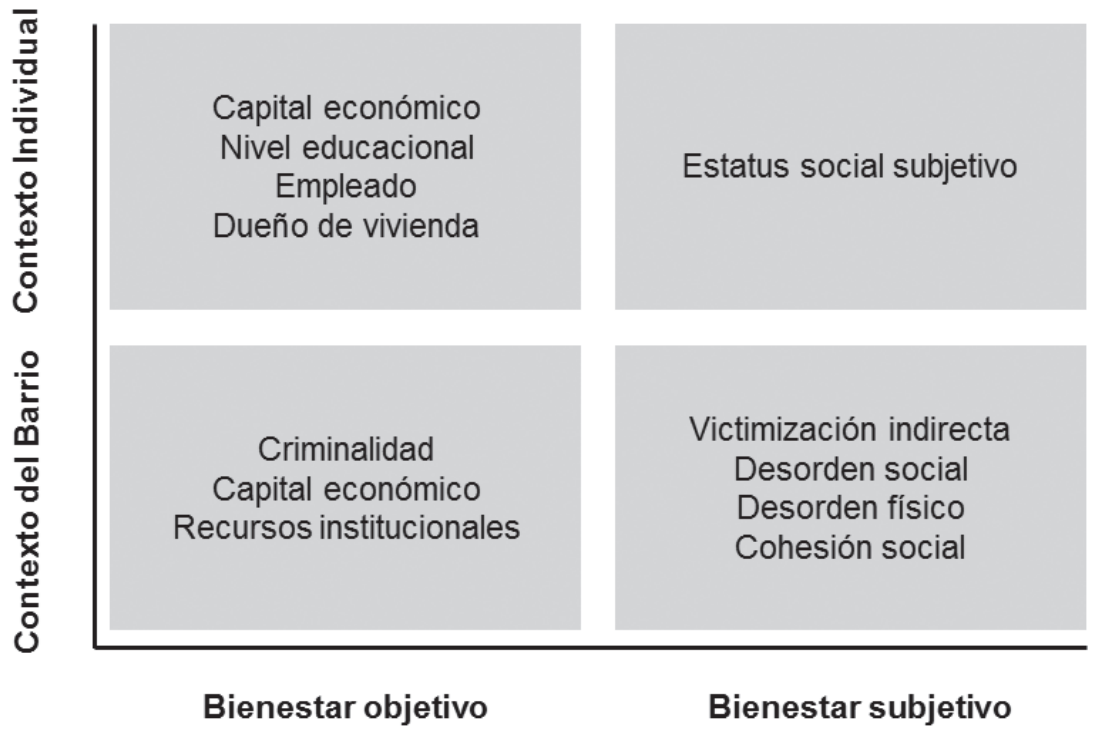

Fuente: Elaboración propia.

\section{Bienestar Objetivo}

Por un lado, en el contexto individual se encuentran las medidas tradicionales para representar el bienestar, como el ingreso, la clase social y los recursos materiales, pero también el nivel educacional y estatus ocupacional. Desde la tesis de la vulnerabilidad comúnmente referida en la literatura en torno a los sentimientos de inseguridad y miedo al crimen, se supone que los grupos más vulnerables expresarían mayores niveles de inseguridad (Hale, 1996; Killias y Clerici, 2000). En la práctica, los autores distinguen entre dos dimensiones de vulnerabilidad: física y social. La primera supone que tanto las mujeres como la tercera edad expresan más miedo al crimen debido a la mayor indefensión que sostienen ante eventuales situaciones de violencia o delincuencia (Pantazis, 2000). Por otro lado, sobre la vulnerabilidad social se sugiere que quienes pertenecen a minorías étnicas o presentan un bajo nivel socioeconómico, así como menores niveles de educación, una situación de desempleo, y menor tenencia de bienes (v.g. casa propia), percibirían mayores niveles de riesgo e inseguridad, especialmente por la falta de capacidad financiera para restablecer los eventuales efectos negativos de tal experiencia (Paydar et al., 2017). 
A propósito de estos mecanismos es que sugerimos un primer conjunto de hipótesis, a saber: si los individuos tienen un mayor capital económico (H1a), nivel educacional (H1b), están empleados (H1c), y son dueños de la vivienda en la que residen (H1d), entonces presentarán un menor grado de inseguridad y temor al crimen.

Por otro lado, en relación con el contexto del barrio, la teoría criminológica se ha enfocado en dilucidar cómo el diseño y estructura de la infraestructura espacial impide o facilita las percepciones de inseguridad. Esta literatura confluye con los denominados "efectos del barrio", enfoque extendido en los estudios urbanos, que ha resaltado la influencia negativa de entornos que muestran una alta segregación de grupos de bajos ingresos, o concentración de la pobreza (van Ham et al., 2012). Por ejemplo, en la estigmatización, los resultados académicos y baja empleabilidad. La hipótesis general supone que las condiciones del barrio producen un efecto que sobrepasa la experiencia individual, y que se materializa a través de algunos mecanismos como la marginación relativa, el desajuste espacial y la exposición a la violencia (Galster, 2012).

Ahora bien, en esta investigación nos alejamos de los componentes más normativos desplegados (v.g. socialización colectiva, efectos de pares), mayormente referidos al contexto norteamericano. Más bien nos concentramos en vincular el sentimiento de inseguridad con procesos de deterioro y decadencia de los barrios, habitualmente representados a partir de la acumulación o sucesión de una serie de fenómenos como la disminución de ingresos, la caída en las tasas de ocupación, la reducción de redes comunitarias y la consumación de delitos violentos (Raleigh y Galster, 2014). Concordamos con que este tipo de fenómenos nos hablan de la retirada del Estado de los sectores geográficos más vulnerables, que, a su vez, emana de la reestructuración neoliberal de la economía (López-Morales, 2016; Slater, 2013). En breve, se traducen en un reforzamiento de la desventaja y desigualdad social, pero que, además, se relacionan con movimientos hacia la re-segregación de las ciudades (Hastings, 2007). Siguiendo la hipótesis general en torno al debate antes mencionado, consideramos que las características objetivas del contexto geográfico se asocian significativamente con los sentimientos de inseguridad y miedo al crimen, más allá de las características individuales. Específicamente, con una mayor frecuencia de criminalidad (H2a), una menor disponibilidad de servicios (H2b), una mayor concentración de la pobreza 
$(\mathrm{H} 2 \mathrm{c})$ y una menor concentración de la riqueza $(\mathrm{H} 2 \mathrm{~d})$ en el entorno geográfico.

\section{Bienestar Subjetivo}

Por un lado, en el contexto individual, el estatus subjetivo refiere a la percepción de una persona respecto de su posición en la estructura social. Por lo general, responde a una creencia que se encuentra disociada de la realidad, en cuanto frecuentemente no se corresponde con los recursos objetivos disponibles (Evans y Kelley, 2004). Según se indica, parece configurarse en función de comparaciones que las personas realizan con sus grupos sociales de referencia (familia, amigos, colegas de trabajo, vecinos) (Castillo et al., 2013), en la que se consideran circunstancias y experiencias pasadas, pero también expectativas futuras y factores psicológicos (Han, 2014). Puesto que el estatus subjetivo es una medida más sensitiva que el capital económico de las personas, es que suponemos que podría evaluar con mayor precisión la influencia acumulada de la posición social y vulnerabilidad, es decir, asociarse negativamente con el miedo al crimen y los sentimientos de inseguridad (H3).

Por otro lado, los mecanismos teóricos que componen lo que entendemos como el bienestar subjetivo en el contexto del barrio, se abordan a través de dimensiones bastante exploradas en la literatura, como las percepciones sobre victimización, el desorden físico y social, pero también una menos enfatizada como es la cohesión social.

Primero, desde la perspectiva de la victimización se supone que los sentimientos de inseguridad y miedo al crimen dentro de una comunidad se producen por el nivel de actividad delictiva, o bien por lo que la gente escucha y percibe sobre la misma en su entorno cercano (Ferraro, 1995; Skogan, 1990). Según se indica, las experiencias indirectas parecen influir incluso más que las experiencias directas y se traducirían en sentimientos de angustia y ansiedad. Se supone que la gente se preocupa aún más cuando es capaz de imaginarse a sí misma como víctima (Hale, 1996). Ahora bien, en esta victimización imaginada también influyen estructuras e instituciones más grandes. Por ejemplo, el escuchar hablar de delincuencia en los medios de comunicación hace más imaginable la victimización, especialmente si la víctima se percibe como alguien similar a la persona referida, o bien si el suceso se produce en situaciones o contextos similares al propio. 
La dramatización y el tono sensacionalista de los medios, así como el énfasis que se da a los delitos más graves dibujarían una opinión pública hacia una sociedad arriesgada y peligrosa. En esta línea, (Garland y Sparks, 2000) hacen referencia al surgimiento de un "complejo de delitos" sobre el cual los ciudadanos se van preocupando y acostumbrando de los temas criminales, manifestando elevados niveles de miedo y ansiedad. Descrito en algunos estudios como el enfoque de la inseguridad generalizada, éste nos habla de cómo estando atrapados en prácticas e instituciones, este complejo exige a los individuos asumir la identidad de víctimas potenciales o reales, y, por consiguiente, pensar, sentir y actuar en consecuencia con ello. En la práctica, esto permite comprender cómo la preocupación por la delincuencia ha penetrado en la conciencia colectiva, entrando en procesos cotidianos, tanto cognitivos como conductuales. Esto también ha sido tratado para el caso Latinoamericano por Carrión y Nuñez-Vega (2006) quien lo describe como "la imaginación o producción social del miedo" que se nutre de diversas fuentes, entre ellas, las estadísticas sobre delitos (o mapas de violencia), los medios de comunicación y también la propia forma de las ciudades y sus divisiones entre los lugares aptos y no aptos para circular. De acuerdo con ello, proponemos la hipótesis de que una experiencia indirecta de victimización se asocia positivamente con el miedo al crimen y los sentimientos de inseguridad (H4a).

Segundo, en relación con el desorden o las llamadas incivilidades, un mecanismo frecuentemente utilizado en la investigación para explicar el sentimiento de inseguridad, enfatiza en el papel que tiene la percepción del entorno, en tanto deterioro físico y social (Hunter, 1978; LaGrange et al., 1992). Teóricamente, Wilson y Kelling (1982) proponen la teoría de las "ventanas rotas", a partir de la cual se postula que estas incivilidades sin resolver impactan en la criminalidad y los sentimientos de temor. Se propone que la inseguridad percibida tiende a ser más común en los barrios que muestran signos de desorden físico como basura y terrenos, edificios, casas y autos abandonados, además de viviendas en mal estado, iluminación escasa y presencia de grafitis (Hinkle, 2014). El desorden social, por su parte, se ha concebido a propósito de prácticas de vandalismo, mendicidad, consumo de drogas, embriaguez pública y prostitución. Teóricamente, el desorden señalaría una ruptura en los autocontroles comunitarios y la unión entre vecinos, además de dar una imagen de que "todo está permitido". En suma, se trata de mecanismos que subrayan preocu- 
paciones que dañan la calidad de vida y el sentido de bienestar de las personas (Jackson y Gray, 2010), y que juegan un papel importante en la decadencia de los barrios (Skogan, 1990). A partir de lo previamente descrito, sugerimos las siguientes hipótesis: cuando los residentes perciben desorden físico $(\mathrm{H} 4 \mathrm{~b})$ y social $(\mathrm{H} 4 \mathrm{c})$, es más probable que estén preocupados sobre la seguridad de su barrio.

Tercero, al explorar la cohesión social como una dimensión del bienestar social, buscamos capturar la calidad de las relaciones entre los miembros de un grupo social, a partir de los sentimientos de apego y compromiso mutuo, junto con la confianza que emerge de valores y normas legitimadas (Berger-Schmitt, 2002; Delhey et al., 2002; Schiefer y Noll, 2016). Se trata de cualidades colectivas, que son características de una sociedad, y que operarían como "the social glue" (Delhey y Dragolov, 2017). Siguiendo a Forrest y Kearns (2001), hablamos de un término que en el contexto barrial reúne a una variedad de aspectos relativos a las formas de interacción social, las cuales se forjarían mediante la participación local y actividad asociativa. Un mecanismo similar, especialmente utilizado en los estudios preocupados por los efectos del barrio, es la eficacia colectiva. Sampson y sus colegas la han definido como la capacidad de una comunidad para mantener una orientación común y así fomentar los controles sociales y la seguridad del entorno (Sampson et al., 1997). Según se ha constatado en la literatura, este mecanismo incide favorablemente en la reducción del desorden, las actividades criminales (Sampson y Raudenbush, 2004), pero también en los sentimientos de inseguridad y miedo al crimen (Brunton-Smith et al., 2014; Donder et al., 2012). Más aún, Jackson (2004) observó que la cohesión social y el control social informal vaticinan las percepciones de riesgo de la misma manera que los niveles de desorden físico y social.

Considerando una operacionalización reciente, concordamos con que la cohesión social tiene tres dimensiones que resultan esenciales (Schiefer y Noll, 2017): las relaciones sociales, donde destacan aspectos como la sociabilidad, confianza y participación; la identidad, que incluye cuestiones como los sentimientos de apego y pertenencia; y las orientaciones hacia el bien común, que implican distintivamente la solidaridad individual e institucional. Sobre esta última, se destacan los sistemas de bienestar social y programas de subvenciones, aspectos que han sido incorporados recientemente en la literatura sobre los sentimientos de inseguridad y miedo al crimen. Por ejemplo, 
a partir de la influencia que tendría la percepción y evaluación de servicios públicos que funcionan en el barrio, como la evaluación de instituciones policiales, educacionales y de salud, pero también de los programas de seguridad municipales (Núñez et al., 2012; Zhao et al., 2015). A partir de lo anterior formulamos un último conjunto de hipótesis de investigación: la sociabilidad (H4d), la confianza en los vecinos (H4e), el apego/arraigo con el barrio (H4f), y la percepción de solidaridad institucional $(\mathrm{H} 4 \mathrm{~g})$, como mecanismos de cohesión social, se asocian negativamente con los sentimientos de inseguridad y miedo al crimen.

\section{EL ESTUDIO}

\section{Datos}

En primer lugar, se utilizan datos primarios que provienen de la Encuesta de Cohesión y Conflicto Barrial desarrollada por el Centro de Estudios de Conflicto y Cohesión Social (COES), a una muestra representativa de 600 residentes adultos localizados en 34 comunas del Área Metropolitana del Gran Santiago (AMGS) de Chile. Las encuestas se realizaron cara a cara en los hogares de las y los residentes. Sobre la base de un muestreo no proporcional estratificado por comuna, la selección de la muestra se llevó a cabo a través de dos etapas. Inicialmente, se escogieron aleatoriamente 150 conglomerados o sectores de viviendas. Posteriormente, se seleccionaron viviendas en cada una de las manzanas. Finalmente, se sondeó a una persona de 18 años o más residente habitual de la vivienda determinada a través de una tabla de Kish. El muestreo fue probabilístico en todas sus etapas, lo que asegura la representatividad de la población de interés. En lo específico, la muestra representa al universo de residentes adultos (mayores de 18 años) de sectores urbanos del AMGS. El error muestral es de $\pm 4,0 \%$ para las estimaciones sobre el total de la muestra, suponiendo un $95 \%$ de confianza y muestreo aleatorio simple. El trabajo de campo se llevó a cabo durante los meses de marzo y abril de 2016 y fue realizado por la consultora Feedback.

En segundo lugar, se incluyen datos georreferenciados por el Centro de Inteligencia Territorial (CIT) respecto de 209 zonas censales, las cuales corresponden a la entidad espacial definida por Instituto Nacional de Estadísticas de Chile (INE) como aquella que más se aproxima a la escala barrial. Se trata de una división urbana formada por un 
conglomerado de manzanas, con la finalidad de facilitar la gestión y administración del censo nacional, y cuya población promedio es de 3.700 personas (Garreton et al., 2016). El levantamiento de la información se obtuvo mediante diversas fuentes: catastro del precenso del 2011: imágenes satelitales actualizadas al 2014; datos administrativos sobre espacios culturales del Consejo Nacional de la Cultura y las Artes al año 2014; información desde las Fichas de Protección Social del Ministerio de Desarrollo Social; y el Censo del año 2002. Dado que muchos de los indicadores se basan en la accesibilidad, se hizo necesaria la actualización de los datos de las redes de transporte al año 2013, con excepción del Gran Santiago que fue corregida al 2014.

\section{Variables}

Nuestra variable dependiente corresponde a un índice promedio que se desprende de 5 ítems referidos, conjuntamente, a los sentimientos de inseguridad y el miedo al crimen. Por un lado, se toman tres ítems de la pregunta que señala, “¿Cuán de acuerdo o en desacuerdo está usted con las siguientes afirmaciones sobre su barrio?". Se utilizó una escala tipo Likert de 5 puntos, desde "muy en desacuerdo" a "muy de acuerdo". Los ítems considerados fueron: "en la noche existe un riesgo de encuentros peligrosos", "las calles son inseguras", y "en este barrio es posible encontrarse con gente mala". Por otro lado, se consideró un ítem que se desprende de la pregunta que indica, "¿Cuán satisfecho está usted con la seguridad del barrio en el que reside?", cuya escala de respuesta de 5 puntos fue desde "muy insatisfecho" a "muy satisfecho". Finalmente, se agregó un ítem en función de la siguiente pregunta: “¿Cuán seguro se siente usted en el barrio donde reside?", cuya escala de respuesta de 5 valores fue desde "muy inseguro" a "muy seguro". La medida que reúne los ítems descritos se respaldó inicialmente en un Análisis de Componentes Principales (ACP), cuya solución se redujo a un solo componente $(\alpha=0.812)$, el cual contiene el $57.5 \%$ de la varianza.

Debe tenerse en cuenta que en este estudio nos enfocamos en construir un indicador compuesto respecto de los sentimientos de inseguridad y miedo al crimen, el cual tiene ventajas y desventajas. Sin duda, nuestra medida compuesta supone mayor robustez en comparación al uso de variables individuales, y por lo tanto los resultados son más confiables. Sin embargo, nuestra medida carece de especificidad en cuanto al tipo de actitud que está representando. Es decir, 
que no permite evaluar detalladamente la sensación de inseguridad, la percepción de riesgo de ser víctima de un crimen, y comparar distintos lugares (v.g. hogar de la persona, vías públicas del barrio donde reside y otros barrios), o distintos períodos del día (día y la noche).

Las variables independientes permiten representar cada una de las dimensiones definidas como bienestar, y se detallan en lo siguiente:

Bienestar objetivo de las personas: lo hemos operacionalizado mediante el capital económico, que corresponde al puntaje ponderado por tenencia de 11 bienes y servicios (v.g. computador, conexión a Internet, cuenta corriente, automóvil); el nivel educacional, que se presenta mediante el formato ISCED (International Standard Classification of Education); el estar empleado a tiempo completo; y ser propietario de la vivienda que habita.

Bienestar subjetivo de las personas: se captura a través de una medida que se toma de la siguiente frase: "Utilizando la siguiente escala, donde 0 es el nivel más bajo y 10 el más alto, ¿en qué posición se ubicaría usted dentro de la estructura social? Aunque existen otras formas de medir el concepto, este tipo de escala permite dar un mayor rango de posibilidades y una mayor comparabilidad" (Castillo et al., 2013).

Bienestar subjetivo del barrio: responde a un conjunto de variables, tradicionales en la literatura. Primero, abordamos la experiencia de victimización indirecta a través de la siguiente pregunta: "Durante los últimos 12 meses, ¿podría indicarme con qué frecuencia se han producido robos o asaltos a personas, casas y/o vehículos en su barrio?". Segundo, la percepción de desorden social responde a un índice promedio de 8 ítems $(\alpha=0.861)$, que se desprende de la pregunta, "¿Podría indicarme con qué frecuencia usted o alguien de su hogar se ha molestado o incomodado por cualquiera de los siguientes problemas con sus vecinos?" Se consideran cuestiones como ruidos molestos, malos olores ocasionados por vecinos y presencia de basura en las calles. Tercero, la percepción de desorden físico se midió a través de un índice promedio de 3 ítems $(\alpha=0.605)$, considerando el siguiente frase: “¿Cuán de acuerdo o en desacuerdo está usted con las siguientes afirmaciones sobre el mantenimiento y cuidado de su barrio?" Los ítems utilizados fueron "hay demasiados hoyos en las calles del barrio", "muchos edificios o casas están en malas condiciones" y "hay muchas paredes o murallas rayadas (grafitis)". 
Cuarto, en relación con las medidas de cohesión social, la sociabilidad se obtiene mediante un índice promedio de 3 ítems ( $\alpha=0.842)$, a través de la pregunta “¿Cuán de acuerdo o en desacuerdo está usted con las siguientes afirmaciones sobre las relaciones sociales de su barrio?" Los ítems sondeados fueron "la gente es cordial", "la gente es sociable" y "es fácil hacer amigos". La confianza responde a la siguiente pregunta: "En términos generales, ¿cuánto confía usted en sus vecinos?". Los sentimientos de apego/arraigo al barrio corresponden a un índice promedio de 4 ítems $(\alpha=0.913)$, utilizando la siguiente pregunta, "¿Cuán de acuerdo o en desacuerdo está usted con las siguientes afirmaciones sobre el apego que usted tiene a su barrio?" Los ítems considerados fueron "este barrio es ideal para mí", "este barrio es parte de mí", "me identifico con la gente de este barrio" y "me siento integrado/a en este barrio". Finalmente, la solidaridad institucional se midió a través de un índice promedio de 4 ítems $(\alpha=0.768)$, sobre la percepción de servicios públicos. En efecto, se captura el grado de acuerdo de los residentes con una adecuada provisión de servicios sociales, carabineros, servicios de salud y servicios para los adultos mayores. Cabe precisar, que las medidas referidas al concepto de cohesión, que adaptamos al contexto del barrio, se extraen de una clasificación reciente desarrollada por Schiefer y Noll (2017), donde se destacan dimensiones como las relaciones sociales, la confianza, e identificación, más que las cuestiones más normativas tradicionalmente consideradas en el debate sociológico.

Bienestar objetivo del barrio: las características objetivas del barrio, definido como la zona censal de residencia, fueron capturadas mediante información georreferenciada. Con el objetivo de reducir los 16 indicadores disponibles del contexto geográfico, utilizamos Análisis de Componentes Principales (ACP), el cual ha sido previamente usado en estudios similares (Jackson y Gray, 2010; Paydar et al., 2017). Este método se basa en la asociación entre variables, cuyo objetivo es optimizar las medidas, pero también evitar problemas de multicolinealidad. Los 4 factores encontrados, claramente consistentes con las teorías revisadas, refieren a la criminalidad, disponibilidad de servicios, concentración de riqueza y concentración de pobreza del barrio. Estos dos últimos factores refieren al capital económico del barrio. En su conjunto, los factores explican el $73 \%$ de la varianza total. La Tabla 1 presenta el análisis realizado. 
Tabla 1

Análisis de Componentes Principales de las Características del Barrio

\begin{tabular}{lcccc}
\hline Factores extraídos & \multicolumn{5}{c}{ Cargas factoriales } \\
\hline Factor 1: Criminalidad & 0.936 & 0.080 & 0.079 & -0.138 \\
Hurtos & 0.904 & 0.086 & 0.130 & 0.236 \\
Lesiones & 0.835 & -0.144 & 0.077 & -0.044 \\
Riñas & 0.832 & -0.128 & 0.283 & 0.086 \\
Seguridad pública & 0.815 & 0.409 & 0.197 & -0.133 \\
Robos & 0.770 & -0.074 & 0.019 & 0.339 \\
Propiedad privada & & & & \\
Factor 2: Concentración de riqueza & -0.080 & 0.805 & -0.059 & 0.110 \\
Concentración de grupos altos & 0.165 & 0.761 & 0.167 & -0.305 \\
Precio del suelo (UF) & -0.005 & 0.693 & 0.081 & -0.431 \\
Puntos promedio & -0.026 & 0.677 & -0.069 & -0.408 \\
Concentración de grupos medios-altos & & & & \\
Factor 3: Disponibilidad de servicios & 0.204 & 0.052 & 0.792 & 0.013 \\
Indicador de servicios públicos & 0.082 & -0.058 & 0.768 & 0.143 \\
Indicador de equipamientos culturales & 0.106 & 0.199 & 0.764 & -0.066 \\
Indicador de equipamientos deportivos públicos & 0.084 & -0.086 & 0.688 & -0.190 \\
Indicador de áreas verdes & & & & \\
Factor 4: Concentración de pobreza. & 0.070 & -0.292 & -0.017 & 0.869 \\
Concentración de grupos bajos & 0.110 & -0.174 & -0.059 & 0.845 \\
Concentración de grupos medios-bajos & 4.957 & 3.646 & 1.980 & 1.103 \\
\hline Valores propios & 30.980 & 22.789 & 12.376 & 6.894 \\
Porcentaje de la varianza explicada & & & & \\
\hline
\end{tabular}

Fuente: Encuesta de Cohesión y Conflicto Barrial COES.

Nota: Los valores en negrita indican el componente en donde las variables tienen cargas más altas.

Es relevante aclarar que las estadísticas oficiales sobre las distintas formas de criminalidad consideradas probablemente se encuentren subrepresentadas, porque no todos los delitos son denunciados por las víctimas. De todas formas, consideramos que sintetizar las distintas formas de criminalidad en un componente, nos permite reducir dicho sesgo en los análisis.

Finalmente, se controla por una serie de medidas comunes en las investigaciones previas: sexo, edad, estado civil, número de niños en el hogar, tipo de vivienda y tiempo de residencia en el barrio. La Tabla 2 muestra los valores y estadísticos descriptivos de cada una de las variables. 
Tabla 2

Operacionalización de Variables y Análisis Descriptivo Univariado

\begin{tabular}{|c|c|c|c|c|c|}
\hline Mecanismos y variables & Categorías & $\begin{array}{c}\text { Media/ } \\
\text { Propor- } \\
\text { ción }\end{array}$ & SD & Min & Max \\
\hline \multicolumn{6}{|l|}{ Variable dependiente } \\
\hline \multicolumn{2}{|c|}{ Sentimientos de inseguridad y miedo al crimen } & 3.12 & 0.76 & 1 & 5 \\
\hline \multicolumn{6}{|l|}{ Variables independientes } \\
\hline \multicolumn{6}{|l|}{ Contexto individual } \\
\hline \multicolumn{6}{|l|}{ Controles } \\
\hline \multirow[t]{2}{*}{ Género } & Femenino & 0.52 & & & \\
\hline & Masculino & 0.48 & & & \\
\hline Edad & Años & 43.80 & 16.44 & 18 & 85 \\
\hline \multirow[t]{2}{*}{ Estado civil } & Casado o conviviendo & 0.51 & & & \\
\hline & Otro & 0.49 & & & \\
\hline Residentes niños & Número de niños & 1.18 & 1.21 & 0 & 4 \\
\hline \multirow[t]{4}{*}{ Tipo de vivienda } & Aislada & 0.28 & & & \\
\hline & Semi pareada & 0.38 & & & \\
\hline & Pareada & 0.25 & & & \\
\hline & Departamento & 0.09 & & & \\
\hline Tiempo de residencia & Años de residencia & 22.44 & 16.01 & 1 & 84 \\
\hline \multicolumn{6}{|l|}{ Bienestar objetivo } \\
\hline Capital económico & $\begin{array}{l}\text { Puntaje ponderado de bienes } \\
\text { y servicios }\end{array}$ & 301.79 & 221.30 & 0 & 1000 \\
\hline Nivel educacional & Formato ISCED & 3.17 & 1.40 & 0 & 6 \\
\hline \multirow[t]{2}{*}{ Estatus ocupacional } & Empleado & 0.65 & & & \\
\hline & Otro & 0.35 & & & \\
\hline \multirow[t]{2}{*}{ Tipo de propietario } & Dueño de la vivienda & 0.65 & & & \\
\hline & Otro & 0.35 & & & \\
\hline \multicolumn{6}{|l|}{ Bienestar subjetivo } \\
\hline Estatus subjetivo & $\begin{array}{l}1 \text { (bajo estatus) - } 10 \text { (alto } \\
\text { status) }\end{array}$ & 4.80 & 1.51 & 0 & 10 \\
\hline \multicolumn{6}{|l|}{ Contexto del barrio } \\
\hline \multicolumn{6}{|l|}{ Bienestar objetivo } \\
\hline Criminalidad & Puntuaciones factoriales & 0.00 & 1.00 & -1 & 9 \\
\hline Concentración de riqueza & Puntuaciones factoriales & 0.00 & 1.00 & -3 & 5 \\
\hline Disponibilidad de servicios & Puntuaciones factoriales & 0.00 & 1.00 & -1 & 7 \\
\hline Concentración de pobreza & Puntuaciones factoriales & 0.00 & 1.00 & -2 & 4 \\
\hline
\end{tabular}


Tabla 2

Operacionalización de Variables y Análisis Descriptivo Univariado (cont.)

\begin{tabular}{|c|c|c|c|c|c|}
\hline Mecanismos y variables & Categorías & $\begin{array}{c}\text { Media/ } \\
\text { Propor- } \\
\text { ción }\end{array}$ & SD & Min & Max \\
\hline \multicolumn{6}{|l|}{ Bienestar subjetivo } \\
\hline Victimización indirecta & 1 (nunca) - 5 (siempre) & 2.60 & 1.24 & 1 & 5 \\
\hline Desorden social & $\begin{array}{l}1 \text { (muy en desacuerdo) - } 5 \\
\text { (muy de acuerdo) }\end{array}$ & 2.02 & 0.88 & 1 & 5 \\
\hline Desorden físico & $\begin{array}{l}1 \text { (muy en desacuerdo) - } 5 \\
\text { (muy de acuerdo) }\end{array}$ & 3.16 & 0.80 & 1 & 5 \\
\hline \multicolumn{6}{|l|}{ Cohesión social } \\
\hline Sociabilidad & $\begin{array}{l}1 \text { (muy insatisfecho) - } 5 \\
\text { (muy satisfecho) }\end{array}$ & 3.27 & 0.82 & 1 & 5 \\
\hline Confianza en vecinos & 1 (nada) - 5 (mucha) & 3.19 & 1.19 & 1 & 5 \\
\hline Apego/arraigo al barrio & $\begin{array}{l}1 \text { (muy en desacuerdo) - } 5 \\
\text { (muy de acuerdo) }\end{array}$ & 3.37 & 0.92 & 1 & 5 \\
\hline Solidaridad institucional & $\begin{array}{l}1 \text { (muy en desacuerdo) - } 5 \\
\text { (muy de acuerdo) }\end{array}$ & 3.00 & 0.77 & 1 & 5 \\
\hline
\end{tabular}

Fuente: Encuesta de Cohesión y Conflicto Barrial COES.

\section{Procedimientos de Análisis}

Dado que la variable dependiente es continua, utilizamos modelos de regresión lineal múltiple. Ahora bien, puesto que los individuos fueron seleccionados aleatoriamente mediante un muestreo estratificado, y están agrupados en 209 zonas censales, utilizamos efectos fijos por comuna, y corregimos por errores estándar robustos estimados por clúster de barrios, con la finalidad de evitar sesgos en algunos coeficientes. Los análisis estadísticos fueron realizados mediante el software Stata 13.

\section{RESULTADOS}

Inicialmente, la Tabla 3 muestra las correlaciones entre algunas variables de interés. Se observa que las mayores asociaciones positivas con los sentimientos de inseguridad y miedo al crimen las sostienen las percepciones de desorden social $(r=0.509)$, y las experiencias de victimización indirecta $(r=0.47)$. En la misma dirección se aprecian las relaciones con las percepciones de desorden físico, pero con un coeficiente más moderado $(r=0.348)$. 
Tabla 3

Análisis de Correlaciones Bivariadas (Coeficiente de Pearson)

\begin{tabular}{lcccccccccc}
\hline & 1 & 2 & 3 & 4 & 5 & 6 & 7 & 8 & 9 & 10 \\
\hline 1 Sentimientos de inseguridad & & & & & & & & & \\
2 Capital económico & -0.249 & & & & & & & & \\
3 Nivel educacional (ISCED) & -0.163 & 0.454 & & & & & & & \\
4 Estatus subjetivo & -0.192 & 0.235 & 0.152 & & & & & & \\
5 Victimización indirecta & 0.470 & -0.096 & -0.081 & -0.101 & & & & & \\
6 Desorden social & 0.509 & -0.126 & -0.061 & -0.158 & 0.394 & & & & \\
7 Desorden físico & 0.348 & -0.230 & -0.121 & -0.035 & 0.203 & 0.317 & & & \\
8 Sociabilidad & -0.193 & -0.030 & -0.009 & 0.103 & -0.076 & -0.156 & -0.048 & & \\
9 Confianza en vecinos & -0.339 & 0.145 & 0.084 & 0.246 & -0.219 & -0.316 & -0.194 & 0.260 & & \\
10 Apego/arraigo al barrio & -0.413 & 0.179 & 0.065 & 0.258 & -0.184 & -0.361 & -0.252 & 0.271 & 0.444 & \\
11 Solidaridad institucional & -0.375 & 0.148 & 0.068 & 0.230 & -0.171 & -0.304 & -0.254 & 0.235 & 0.240 & 0.348 \\
\hline Fuente: Encuesta de Cohesión y Conflicto Barrial COES. & & & & & \\
Nota: Todos los coeficientes son significativos (p<.05) menos aquellos destacados en negrita.
\end{tabular}

Por otro lado, las correlaciones negativas más fuertes con nuestra medida de interés se aprecian en el apego/arraigo al barrio $(r=-0.413)$ y las percepciones de solidaridad institucional $(r=0.375)$. Todos estos son aspectos que representan el bienestar subjetivo en el contexto barrial. Por su parte, las medidas objetivas del contexto individual, aunque presentan una dirección coherente con la teoría de la vulnerabilidad, sostienen correlaciones más pequeñas comparativamente. El coeficiente de asociación más alto lo alcanza el capital económico $(r=-0.249)$. En la práctica, se trata de hallazgos consistentes con la evidencia disponible. Cabe precisar que las correlaciones entre variables independientes parecen bastante moderadas, con la excepción de asociación positiva que se evidencia entre el apego/arraigo al barrio y la confianza en vecinos $(r=-0.444)$. Esto despeja algunas dudas respecto de eventuales problemas de multicolinealidad.

La Tabla 4 muestra los resultados de nueve modelos de regresión lineal múltiple, con efectos fijos por comuna y errores clúster estandarizados por barrio, con la finalidad de estimar los sentimientos de inseguridad y miedo al crimen. La tabla presenta los coeficientes beta, errores de estimación (entre paréntesis) y los niveles de significación para cada variable. El modelo 1 incluye una serie de variables de control, como sexo y edad, las cuales se mantienen durante todas estimaciones; el modelo 2 agrega las medidas de bienestar objetivo y subjetivo en el contexto individual; el modelo 3 adiciona el 
bienestar objetivo del contexto barrial (criminalidad, disponibilidad de servicios, concentración de pobreza/riqueza); el modelo 4 adosa las experiencias de victimización indirecta; el modelo 5 excluye los predictores del bienestar en el contexto individual; el modelo 6 añade las percepciones de desorden (físico y social); el modelo 7 contiene sólo las variables del contexto barrial; el modelo 8 prescinde de las percepciones de desorden físico y social; $y$, finalmente, en el modelo 9 se utilizan todas las medidas consideradas en el estudio.

Tal como se observa en la Tabla 4, el modelo 1 tiene como predictores significativos, tanto al sexo como al número de residentes niños. Ser mujer se asocia con mayores sentimientos de inseguridad y miedo al crimen, respecto de ser hombre. Lo mismo ocurre al incrementarse la cantidad de niños en el hogar. En el modelo 2 se agregan las variables objetivas y subjetivas del contexto individual, las que permiten una mejora respecto del modelo anterior (R-cuadrado ajustado se incrementa a $18.5 \%$ ). En cuanto a la dimensión objetiva, sólo el capital económico tiene una asociación negativa y estadísticamente significativa con el problema de interés. Lo mismo ocurre con el estatus subjetivo. En la primera medida ello ocurre al $99 \%$ de confianza, mientras que en la segunda sólo al 90\%. Al agregar las variables objetivas del contexto barrial en el modelo 3 , tanto la disponibilidad de servicios como la concentración de pobreza se constatan como estimadores estadísticamente significativos, aunque este último sólo al 90\% de confianza. No obstante, la dimensión objetiva del barrio no produce cambios en los otros predictores, permite mejorar levemente el ajuste del modelo (R-cuadrado ajustado se incrementa a 19.2\%).

El modelo 4 adiciona por primera vez las experiencias de victimización indirecta, aspecto definido como parte del bienestar subjetivo en el contexto del barrio. Como era de esperarse, se trata de una medida que muestra una fuerte asociación estadística con nuestra variable de interés. En efecto, un incremento en la frecuencia declarada de robos o asaltos a personas, casas y/o vehículos en el barrio (en los últimos 12 meses), se asocia positivamente con los sentimientos de inseguridad y miedo al crimen. En concordancia con la literatura revisada (Hale, 1996), las experiencias de victimización indirecta parecen tener una influencia mucho mayor que las tasas de criminalidad objetivas relativas al contexto barrial. En nuestro caso hablamos de un predictor que, además, desestima la influencia y significancia estadística de la disponibilidad objetiva de servicios en la zona censal. En la práctica, 
el impacto de este predictor también disminuye el impacto de otras variables como ser mujer, al mismo tiempo que desestima la significancia estadística del estatus subjetivo. Ahora bien, parece interesante constatar que la concentración de pobreza se mantiene como un factor positivo y estadísticamente significativo de los sentimientos de inseguridad y miedo al crimen. En la práctica, las experiencias de victimización indirecta permiten optimizar considerablemente el ajuste del modelo, alcanzando un R-cuadrado de 34\%.

Dada la importancia de este último predictor, en el modelo 5 sólo se incluyen el bienestar objetivo del barrio y las experiencias indirectas de victimización. En esta estimación la concentración de pobreza mejora su coeficiente y significancia estadística, ahora al 95\% de confianza. Esto nos indicaría que el bienestar individual, especialmente los recursos materiales individuales, captura buena parte del impacto ejercido por las características objetivas del barrio, tal y como ha sido constatado en otras investigaciones. Sin embargo, también queda en evidencia su poca importancia relativa, en cuanto el R-cuadrado sólo disminuye levemente al $33.3 \%$.

Tabla 4

Modelos de Regresión Lineal Múltiple de los Sentimientos de Inseguridad y Miedo al Crimen

\begin{tabular}{|c|c|c|c|c|c|c|c|c|c|}
\hline & Modelo1 & Modelo 2 & Modelo 3 & Modelo 4 & Modelo 5 & Modelo 6 & Modelo 7 & Modelo 8 & Modelo 9 \\
\hline \multicolumn{10}{|l|}{ Contexto individual } \\
\hline \multicolumn{10}{|l|}{ Controles } \\
\hline \multirow[t]{2}{*}{ Género=mujer } & $0.206^{* * *}$ & $0.195^{* * *}$ & $0.203^{* * *}$ & $0.131^{* *}$ & $0.143^{* * *}$ & $0.135^{* * *}$ & $0.115^{* *}$ & $0.098^{*}$ & $0.103^{* *}$ \\
\hline & $(0.056)$ & $(0.057)$ & $(0.057)$ & $(0.052)$ & $(0.052)$ & $(0.048)$ & $(0.049)$ & $(0.051)$ & $(0.049)$ \\
\hline \multirow[t]{2}{*}{ Edad (años) } & -0.002 & -0.003 & -0.002 & -0.000 & 0.000 & 0.002 & $0.004^{* *}$ & 0.002 & 0.003 \\
\hline & $(0.002)$ & $(0.003)$ & $(0.003)$ & $(0.002)$ & $(0.002)$ & $(0.002)$ & $(0.002)$ & $(0.002)$ & $(0.002)$ \\
\hline \multirow[t]{2}{*}{ Estado civil=soltero } & 0.106 & 0.102 & 0.105 & 0.086 & 0.093 & 0.050 & 0.052 & 0.074 & 0.050 \\
\hline & $(0.071)$ & $(0.071)$ & $(0.071)$ & $(0.065)$ & $(0.064)$ & $(0.061)$ & $(0.060)$ & $(0.062)$ & $(0.061)$ \\
\hline \multirow{2}{*}{$\begin{array}{l}\text { Número de residentes } \\
\text { niños }\end{array}$} & $0.050^{* *}$ & $0.045^{*}$ & $0.046^{*}$ & $0.041^{*}$ & $0.042^{*}$ & $0.041^{*}$ & 0.034 & 0.034 & 0.035 \\
\hline & $(0.025)$ & $(0.025)$ & $(0.025)$ & $(0.023)$ & $(0.023)$ & $(0.022)$ & $(0.022)$ & $(0.022)$ & $(0.021)$ \\
\hline \multicolumn{10}{|l|}{$\begin{array}{l}\text { Tipo de vivienda } \\
\text { (ref=aislada) }\end{array}$} \\
\hline \multirow[t]{2}{*}{ Semi pareada } & -0.079 & -0.083 & -0.101 & -0.061 & -0.059 & -0.074 & -0.097 & -0.096 & -0.099 \\
\hline & $(0.082)$ & $(0.081)$ & $(0.081)$ & $(0.073)$ & (0.073) & $(0.068)$ & $(0.067)$ & $(0.069)$ & $(0.066)$ \\
\hline \multirow[t]{2}{*}{ Pareada } & 0.062 & 0.025 & 0.013 & 0.015 & 0.035 & -0.005 & -0.066 & -0.069 & -0.077 \\
\hline & (0.113) & (0.114) & (0.111) & $(0.094)$ & (0.092) & $(0.086)$ & $(0.086)$ & $(0.091)$ & $(0.086)$ \\
\hline
\end{tabular}


Tabla 4

Modelos de Regresión Lineal Múltiple de los Sentimientos de Inseguridad y Miedo al Crimen (Cont.)

\begin{tabular}{|c|c|c|c|c|c|c|c|c|c|}
\hline & Modelo1 & Modelo 2 & Modelo 3 & Modelo 4 & Modelo & Modelo 6 & Modelo 7 & Mo & \\
\hline \multirow[t]{2}{*}{ Departamento } & 0.137 & 0.112 & 0.100 & 0.128 & 0.139 & 0.043 & 0.039 & 0.091 & 0.034 \\
\hline & $(0.146)$ & $(0.148)$ & $(0.148)$ & $(0.121)$ & $(0.121)$ & $(0.110)$ & (0.114) & $(0.122)$ & $(0.111)$ \\
\hline \multirow{2}{*}{$\begin{array}{l}\text { Años de residencia en } \\
\text { el barrio }\end{array}$} & -0.009 & -0.013 & -0.016 & -0.011 & -0.008 & -0.017 & -0.001 & 0.007 & -0.002 \\
\hline & $(0.024)$ & $(0.025)$ & $(0.026)$ & $(0.022)$ & $(0.021)$ & $(0.019)$ & $(0.020)$ & $(0.021)$ & $(0.021)$ \\
\hline \multicolumn{10}{|l|}{ Bienestar objetivo } \\
\hline \multirow[t]{2}{*}{ Capital económico } & & $-0.000^{* * *}$ & $-0.000^{* *}$ & $-0.000 * * *$ & & & & $-0.000^{* *}$ & $-0.000^{* *}$ \\
\hline & & $(0.000)$ & $(0.000)$ & $(0.000)$ & & & & $(0.000)$ & $(0.000)$ \\
\hline \multirow[t]{2}{*}{$\begin{array}{l}\text { Nivel educacional } \\
\text { (ISCED) }\end{array}$} & & -0.019 & -0.008 & 0.004 & & & & 0.001 & 0.001 \\
\hline & & $(0.025)$ & $(0.025)$ & $(0.023)$ & & & & $(0.024)$ & $(0.023)$ \\
\hline \multirow{2}{*}{$\begin{array}{l}\text { Estatus } \\
\text { ocupacional=empleado }\end{array}$} & & 0.086 & 0.070 & 0.048 & & & & 0.035 & 0.040 \\
\hline & & $(0.062)$ & $(0.063)$ & $(0.057)$ & & & & $(0.054)$ & $(0.055)$ \\
\hline \multirow{2}{*}{$\begin{array}{l}\text { Tipo de } \\
\text { propietario=dueño }\end{array}$} & & 0.050 & 0.056 & 0.058 & & & & 0.077 & 0.061 \\
\hline & & $(0.069)$ & $(0.069)$ & $(0.067)$ & & & & $(0.059)$ & $(0.059)$ \\
\hline \multicolumn{10}{|l|}{ Bienestar subjetivo } \\
\hline \multirow[t]{2}{*}{ Estatus subjetivo } & & $-0.041^{*}$ & $-0.040^{*}$ & -0.026 & & & & 0.015 & 0.013 \\
\hline & & $(0.024)$ & $(0.024)$ & $(0.022)$ & & & & $(0.020)$ & $(0.019)$ \\
\hline \multicolumn{10}{|l|}{ Contexto del barrio } \\
\hline \multicolumn{10}{|l|}{ Bienestar objetivo } \\
\hline \multirow[t]{2}{*}{ Criminalidad } & & & 0.015 & 0.023 & 0.026 & 0.028 & 0.016 & 0.006 & 0.012 \\
\hline & & & $(0.030)$ & $(0.023)$ & $(0.025)$ & $(0.028)$ & $(0.027)$ & $(0.025)$ & $(0.027)$ \\
\hline \multirow[t]{2}{*}{ Concentración de riqueza } & & & -0.027 & -0.053 & -0.075 & -0.060 & -0.046 & -0.035 & -0.033 \\
\hline & & & $(0.082)$ & $(0.070)$ & $(0.073)$ & $(0.064)$ & $(0.058)$ & $(0.061)$ & $(0.058)$ \\
\hline \multirow[t]{2}{*}{$\begin{array}{l}\text { Disponibilidad de } \\
\text { servicios }\end{array}$} & & & $-0.070^{* *}$ & -0.016 & -0.021 & -0.004 & -0.008 & -0.014 & -0.004 \\
\hline & & & $(0.029)$ & $(0.026)$ & $(0.026)$ & $(0.022)$ & $(0.023)$ & $(0.026)$ & $(0.023)$ \\
\hline \multirow[t]{2}{*}{$\begin{array}{l}\text { Concentración de } \\
\text { pobreza }\end{array}$} & & & $0.075^{*}$ & $0.066^{*}$ & $0.081^{* *}$ & 0.051 & 0.044 & 0.051 & 0.037 \\
\hline & & & $(0.044)$ & $(0.036)$ & $(0.036)$ & $(0.032)$ & $(0.031)$ & $(0.034)$ & $(0.032)$ \\
\hline \multicolumn{10}{|l|}{ Bienestar subjetivo } \\
\hline \multirow[t]{2}{*}{ Victimización indirecta } & & & & $0.264^{* * *}$ & $0.267^{* * *}$ & $0.211^{* * *}$ & $0.206^{* * *}$ & $0.237^{* * *}$ & $0.207^{* * *}$ \\
\hline & & & & $(0.026)$ & $(0.026)$ & $(0.027)$ & $(0.027)$ & $(0.027)$ & $(0.027)$ \\
\hline \multirow[t]{2}{*}{ Desorden social } & & & & & & $0.236^{* * *}$ & $0.164^{* * *}$ & & $0.165^{* * *}$ \\
\hline & & & & & & $(0.038)$ & $(0.039)$ & & $(0.039)$ \\
\hline \multirow[t]{2}{*}{ Desorden físico } & & & & & & $0.163^{* * *}$ & $0.118^{* * *}$ & & $0.110^{* * *}$ \\
\hline & & & & & & $(0.035)$ & $(0.034)$ & & $(0.034)$ \\
\hline
\end{tabular}


Tabla 4

Modelos de Regresión Lineal Múltiple de los Sentimientos de Inseguridad y Miedo al Crimen (Cont.)

\begin{tabular}{|c|c|c|c|c|c|c|c|c|c|}
\hline & Modelo 1 & Modelo 2 & Modelo3 & Modelo 4 & Modelo 5 & Modelo 6 & Modelo 7 & 7 Modelo 8 & Modelo 9 \\
\hline \multicolumn{10}{|l|}{ Cohesión social } \\
\hline \multirow[t]{2}{*}{ Sociabilidad } & & & & & & & -0.002 & -0.008 & -0.002 \\
\hline & & & & & & & $(0.037)$ & $(0.037)$ & $(0.036)$ \\
\hline \multirow[t]{2}{*}{ Confianza en vecinos } & & & & & & & $-0.048^{*}$ & $-0.070 * * *$ & $-0.046^{*}$ \\
\hline & & & & & & & $(0.024)$ & $(0.025)$ & $(0.025)$ \\
\hline \multirow{2}{*}{$\begin{array}{l}\text { Apego/arraigo al } \\
\text { barrio }\end{array}$} & & & & & & & $-0.156^{* * *}$ & $*-0.201 * * *$ & $-0.160^{* * *}$ \\
\hline & & & & & & & $(0.032)$ & $(0.034)$ & $(0.033)$ \\
\hline \multirow{2}{*}{$\begin{array}{l}\text { Solidaridad } \\
\text { institucional }\end{array}$} & & & & & & & $-0.105^{* * *}$ & $-0.131^{* * * *}$ & $-0.107^{* * *}$ \\
\hline & & & & & & & $(0.040)$ & $(0.042)$ & $(0.040)$ \\
\hline \multirow[t]{2}{*}{ Constante } & $3.142^{* * *}$ & $3.626^{* * *}$ & $3.562^{* * *}$ & $2.558^{* * *}$ & $2.273^{* * *}$ & $1.264^{* * *}$ & $2.677^{* * *}$ & $3.800^{* * * *}$ & $2.740^{* * * *}$ \\
\hline & $(0.190)$ & $(0.274)$ & $(0.278)$ & $(0.244)$ & $(0.176)$ & $(0.214)$ & $(0.299)$ & $(0.304)$ & $(0.317)$ \\
\hline Observaciones & 600 & 600 & 600 & 600 & 600 & 600 & 600 & 600 & 600 \\
\hline R-cuadrado ajustado & 0.171 & 0.185 & 0.192 & 0.340 & 0.333 & 0.422 & 0.471 & 0.436 & 0.472 \\
\hline
\end{tabular}

En el modelo 6 se constata el valor de la dimensión subjetiva del contexto barrial para explicar los sentimientos de inseguridad y miedo al crimen. En efecto, aunque hasta ahora la concentración de pobreza, como medida objetiva del contexto barrial, se había conservado como predictor significativo de nuestra variable de interés, tal situación se desestima al incluir el desorden percibido. En la práctica, las percepciones de los residentes sobre el desorden físico y social presentan coeficientes positivos y significativos al $99 \%$ de confianza, al mismo tiempo que producen una mejora sustancial en el ajuste del modelo, elevando el R-cuadrado a $42.2 \%$. Este es un primer indicio de que el bienestar subjetivo parece ser mucho más importante como predictor de inseguridad que el bienestar objetivo.

Al adicionar la cohesión social del barrio en el modelo 7, en función de las declaraciones de los residentes, es posible constatar que un mayor apego/arraigo al barrio, las percepciones de solidaridad institucional y la confianza en vecinos se asociande forma negativa y significativa con los sentimientos de inseguridad y miedo al crimen. Aunque para el último predictor esto ocurre sólo al $90 \%$ de confianza. Adicionalmente, el ajuste del modelo muestra un incremento nada despreciable 
(R-cuadrado ajustado se incrementa a 47.1\%). Al comparar con el modelo anterior, la agregación de estos últimos predictoresreduce la influencia de las otras variables subjetivas del contexto barrial, especialmente del desorden físico y social, al mismo tiempo que confirma que la dimensión subjetiva del barrio, y mayormente las experiencias de victimización, es más relevante que la objetiva.

El modelo 8 excluye las percepciones de desorden físico y social, ejercicio que permite rescatar algunos matices respecto de la importancia de la cohesión barrial. En efecto, podemos ver que, a diferencia del modelo anterior, la confianza en vecinos se muestra significativo al $99 \%$ de confianza. Asimismo, al comparar con el modelo 6 es posible inferir, con mesura, que la cohesión en su conjunto explica levemente más que las percepciones de desorden. En la práctica, este modelo alcanza un R-cuadrado de $43.6 \%$.

Nuestra última estimación (modelo 9) incorpora todas las variables de estudio simultáneamente, con lo cual se logra explicar el $47.2 \%$ de la varianza de los sentimientos de inseguridad y miedo al crimen. Dado que algunos predictores podrían estar correlacionados (véase Tabla 3), hemos realizado pruebas de multicolinealidad para evitar los problemas subyacentes. En efecto, los valores del factor de inflación de la varianza (FIV) calculados nos permiten continuar con tranquilidad. En la práctica, ningún estadístico supera el 2.5 recomendado. Tal como se aprecia en la Tabla 4, nuestros resultados son similares a los que usualmente se reportan en la literatura en el área, es decir, que las experiencias de victimización indirecta, junto con las percepciones de desorden físico y social explican buena parte de la varianza asociada a los sentimientos de inseguridad y miedo al crimen. Asimismo, que los aspectos objetivos individuales y del barrio no parecen ser tan significativos, o bien son capturados por las configuraciones más subjetivas. Sólo se observan asociaciones estadísticamente significativas relativas a ser mujer, además del capital económico individual. Finalmente, se rescata la relevancia del apego/arraigo, la solidaridad institucional y confianza en vecinos como predictores negativos y significativos, los cuales podrían dar cuenta de que, pese a la vulnerabilidad y las disposiciones adversas sobre las características del barrio que se encuentran justificada o injustificadamente en el imaginario de las personas, podría ser viable promover mecanismos de resistencia para atender a los sentimientos de inseguridad generalizados. 


\section{DISCUSIÓN Y CONCLUSIONES}

Esta investigación tuvo como propósito estudiar los sentimientos de inseguridad y miedo al crimen en un contexto distintivo y novedoso, que combina la alta desigualdad con la segregación geográfica, como es la ciudad de Santiago de Chile. La utilización de una muestra representativa de la población de estudio permitió reportar una evidencia más sistemática que complementa aquella informada sobre entornos residenciales desaventajados, a saber: el miedo al crimen en cuanto experiencia indirecta (de la que se sabe, se escucha, frente a la cual se tiene exposición indirecta) juega un papel clave, ya sea en contextos desventajados o privilegiados.

Se testearon múltiples mecanismos teóricos reunidos en el debate, los cuales integramos bajo el concepto de bienestar. Este ejercicio conceptual nos ayudó a determinar con mayor claridad los factores que influyen en las percepciones de interés. Inicialmente, los resultados muestran que la edad, el número de residentes niños, tipo de vivienda, estado civil y los años de residencia en el barrio no tienen relación con los sentimientos de inseguridad y miedo al crimen, luego de controlar por variables teóricamente relevantes. Sobre el sexo de las personas, ser mujer aparece como predictor significativo, pero con cierta cautela. En general, se trata de evidencia coherente con la literatura disponible (Hummelsheim et al., 2011; Pantazis, 2000; Schafer et al., 2006; Vilalta, 2011).

En línea con otros estudios (Kanan y Pruitt, 2002; Wyant, 2008), los hallazgos informan sobre la preponderancia del bienestar subjetivo en el contexto barrial, en la explicación del sentimiento de inseguridad y miedo al crimen, junto con la desestimación de la importancia de variables objetivas tanto en el contexto individual como barrial. Ciertamente los sentimientos de inseguridad y miedo al crimen parecen tener una autonomía relativa respecto de las tasas de delincuencia (Breetzke y Pearson, 2014; Brunton-Smith y Sturgis, 2011), y por ende, recogen otros miedos e inseguridades de los residentes (Amerio y Roccato, 2005; Hale, 1996; Hummelsheim et al., 2011). En efecto, sólo podemos ratificar la influencia del capital económico de las personas (H1a), que habíamos sugerido en nuestro primer conjunto de hipótesis, aunque con un impacto bastante pequeño en comparación con el resto de los predictores estadísticamente significativos. En relación con la dimensión objetiva del bienestar en el contexto del barrio, no encontramos evidencia para validar la serie de hipótesis propuestas (H2), pese a que la concentración de pobreza demostró tener una importancia relativa antes de adicionar las percep- 
ciones de desorden físico y social. Esta última evidencia es sumamente relevante, tomando en cuenta que los datos georreferenciados utilizados nos permitieron evitar problemas de endogeneidad.

En cuanto al bienestar subjetivo en el contexto individual, nuestros resultados revelan que se trata de una dimensión relevante, pero que pierde significancia cuando se incorporan las experiencias indirectas de victimización previa. Por lo tanto, no podemos confirmar la hipótesis planteada al respecto $(\mathrm{H} 3)$. En la práctica, el predictor de victimización, junto con las percepciones de desorden físico y social, capturan buena parte de la explicación sobre los sentimientos de inseguridad y miedo al crimen. Se trata de evidencia robusta y en línea con lo reportado largamente (Breetzke y Pearson, 2014; Hinkle, 2014; McNeeley y Yuan, 2017; Wyant, 2008; Zhao et al., 2015). En efecto, su extensión se asocia positivamente con dichas actitudes, lo que corrobora las tres hipótesis planteadas inicialmente ( $\mathrm{H} 4 \mathrm{a}, \mathrm{H} 4 \mathrm{~b}$ y H4c).

Un reporte novedoso representa la influencia negativa de las variables de cohesión barrial, las cuales han sido discutidas ampliamente en la literatura, mayormente en torno los conceptos de eficacia colectiva y capital social (Brunton-Smith et al., 2014; Sampson et al., 1997). En esta investigación hemos contribuido a mejorar su definición y operacionalización, a partir de aportes recientes (Schiefer y Noll, 2017), y nos hemos encontrado con hallazgos interesantes.

Se constata el aporte limitado de la sociabilidad declarada por los residentes, condición que nos impide hacernos cargo de la hipótesis planteada en un comienzo (H4d). Contrariamente, por un lado, reportamos el aporte de confianza en vecinos, aunque con precaución (H4e). Esto porque buena parte de su importancia se pierde cuando se ingresa a los modelos conjuntamente con las percepciones de desorden físico y social. Por otro lado, confirmamos que el apego/arraigo al barrio (sentido de pertenencia, identificación), es un factor bastante estable y crucial para reducir las percepciones de inseguridad y miedo al crimen (H4f), en concordancia con algunos estudios (Brunton-Smith et al., 2014; Jackson, 2004), y a diferencia de otros (Donder et al., 2012). Lo mismo ocurre con la solidaridad institucional, definida como la percepción de servicios públicos disponibles, donde también ratificamos nuestro supuesto $(\mathrm{H} 4 \mathrm{~g})$.

En concordancia con estudios anteriores, los indicadores de cohesión barrial permiten amortiguar el impacto de las incivilidades, aunque su 
aporte es limitado para restringir el impacto de las experiencias de victimización indirecta. Así pues, podríamos concordar con la afirmación que sostiene que la cohesión barrial, el desorden físico y social y la inseguridad percibida forman parte de un ciclo recíproco (Hawdon et al., 2014). Ahora bien, igualmente hay que tener en cuenta que las distintas medidas que capturan la cohesión social de los barrios podrían operar como mecanismos de resistencia, al mismo tiempo que representar los efectos de la fragmentación comunitaria (Pearson et al., 2015).

Concluimos respaldando la idea de que la protección social provista por el Estado puede atenuar el desarrollo de un temor generalizado de la criminalidad, mediante el aumento de la auto-eficacia, y así también mitigar los diversos miedos sociales y económicos que tiene la gente (Hummelsheim et al., 2011). En parte, también invita a preguntarnos por las dinámicas históricas de los contextos de estudio, y cómo su influencia podría complementar las explicaciones sobre las actitudes en cuestión. No podemos olvidar que la percepción de inseguridad constituye un mecanismo exitoso de la política y las campañas electorales, especialmente en contextos como Santiago de Chile, donde hay una historia reciente de debilitamiento del tejido social bajo el uso de la violencia política, que sólo fue recuperado parcialmente desde lo local y con un marcado sello pragmático y apolítico de las autoridades locales. En efecto, la literatura que aborda este caso muestra cómo el temor a la delincuencia terminó por instalarse como una herramienta de gubermentalidad (Valdivia et al, 2012), provocando que quienes buscaban el poder a nivel local se distanciasen de la política tradicional, optando por centrarse en la solución de "problemas concretos". En efecto, cabe reflexionar sobre la efectividad de medidas orientadas a reducir los sentimientos de inseguridad, cuando las instituciones políticas y los medios de comunicación, generalmente alineados con los intereses de los grupos dominantes, se han establecido como productores de bienestar subjetivo, el cual parece tener mayor fuerza que la propia experiencia directa transversalmente en habitantes de distintos sectores y clases sociales. La economía política de la representación del miedo al crimen (Carrión y Nuñez-Vega, 2006) pareciera ser demasiado efectiva y demasiado eficaz como para entregarla al escrutinio público. Con este artículo esperamos mostrar evidencia útil para abrir esta caja negra.

(Recebido para publicação em 19 de fevereiro de 2017)

(Reapresentado em 6 de Maio de 2019)

(Aprovado para publicação em 12 de julho de 2019) 


\section{REFERENCIAS}

AMERIO, Piero; ROCCATO, Michele. (2005), “A predictive model for psychological reactions to crime in Italy: an analysis of fear of crime and concern about crime as a social problem". Journal of Community E Applied Social Psychology, v. 15, n. 1, pp. 17-28.

BAUMAN, Zigmunt. (2000), Liquid modernity. Cambridge, Polity.

BECK, Ulrich. (1999), World risk society. Cambridge, Polity.

BERGER-SCHMITT, Regina. (2002), "Considering social cohesion in quality of life assessments: concept and measurement". Social Indicators Research, v. 11, pp. 403-428.

BERGMAN, Marcelo; FLOM, Hernán. (2008), “Policía y comunidad: una comparación entre fuerzas policiales del Distrito Federal y Estado de México y la Ciudad Autónoma de Buenos Aires". Documentos de Trabajo del Centro de Investigación y Docencia Económicas (CIDE), n. 33.

BREETZKE, Gregory; PEARSON, Amber. (2014), "The fear factor: examining the spatial variability of recorded crime on the fear of crime". Applied Geography, v. 46, n. 1, pp. 45-52.

BRUNTON-SMITH, Ian; JACKSON, Jonathan; SUTHERLAND, Alex. (2014), "Bridging structure and perception on the neighbourhood ecology of beliefs and worries about violent crime". British Journal of Criminology, v. 54, n. 4, pp. 503-526.

BRUNTON-SMITH, Ian; STURGIS, Patrick. (2011), "Do neighborhoods generate fear of crime? An empirical test using the British Crime Survey". Criminology, v. 49, n. 2, pp. 331-369.

CALDEIRA, Teresa. (2000), City of walls: crime, segregation and citizenship in Sao Paulo. Berkeley, University of California Press.

CARRIÓN, Fernando; NÚÑEZ-VEGA, Jorge. (2006), "La inseguridad en la ciudad: hacia una comprensión de la producción social del miedo". EURE, v. 32, n. 97, pp. 7-16.

CASTILLO, Juan Carlos; MIRANDA, Daniel; MADERO, Ignacio. (2013), “Todos somos de clase media: sobre el estatus social subjetivo en Chile". Latin American Research Review, v. 48 , n. 1 , pp. $155-173$.

CHEVIGNY, Paul. (2003). "The populism of fear: politics of crime in the Americas". Punishment $\mathcal{E}$ Society, v. 5, n. 1, pp. 77-96.

CORRADI, Juan; WEISS, Patricia; GARRETÓN, Manuel Antonio (eds.). (1992), Fear at the edge: state terror and resistance in Latin America. Berkeley, University of California Press.

DAMMERT, Lucía; ARIAS, Patricia. (2007), “El desafío de la delincuencia en América Latina: diagnóstico y respuestas de política”. in L. Dammert; P. Arias (eds.), Seguridad y violencia: desafíos para la ciudadanía. Santiago, Facultad Latinoamericana de Ciencias Sociales, FLACSO-Chile, pp. 21-66.

DAMMERT, Lucía; MALONE, Mary. (2003), "Fear of crime or fear of life? public insecurities in chile". Bulletin of Latin American Research, v. 22, n. 1, pp. 79-101. 
DAMMERT, Lucía; MALONE, Mary. (2006). "Does it take a village? policing strategies and fear of crime in Latin America". Latin American Politics and Society, v. 48, n. 4, pp. 27-51.

DAMMERT, Lucía. (2012). Fear and crime in latin america: redefining state-society relations. New York, Routledge.

DELHEY, Jan; BÖHNKE, Petra; HABICH, Roland; ZAPF, Wolfgang. (2002), “Quality of life in a European perspective: the EUROMODULE as a new instrument for comparative Welfare Research". Social Indicators Research, v. 58, n. 1-3, pp. 161-175.

DELHEY, Jan; DRAGOLOV, Georgi. (2016). “Happier together. social cohesion and subjective well-being in Europe". International Journal of Psychology, v. 51, n. 3, pp. 163-176.

DONDER, Liesbeth et al. (2012), "Social capital and feelings of unsafety in later life: a study on the influence of social networks, place attachment, and civic participation on perceived safety in Belgium". Research on Aging, v. 34, n. 4, pp. 425-448.

EVANS, Mariah; KELLEY, Jonathan. (2004), "Subjective social location: data from 21 nations". International Journal of Public Opinion Research, v. 16, n. 1, pp. 3-38.

FERRARO, Kenneth. (1995), Fear of crime: interpreting victimization risk. Albany, SUNY Press.

FORREST, Ray; KEARNS, Ade. (2001), "Social cohesion, social capital and the neighbourhood". Urban Studies, v. 38, n. 12, pp. 2125-2143.

FUREDI, Frank. (2002), The culture of fear. London, Cassell.

GALSTER, George. (2012), "The mechanism(s) of neighbourhood effects: theory, evidence, and policy implications". in M. Van et al. (eds.), Neighbourhood effects research: new perspectives. Netherlands, Springer, pp. 23-56.

GARLAND, David; SPARKS, Richard. (2000), "Criminology, social theory and the challenge of our times". The British Journal of Criminology, v. 40, n. 2, pp. 189-204.

GAROFALO, James. (1981), "The fear of crime: causes and consequences". The Journal of Criminal Law and Criminology, v. 72, n. 2, pp. 839-857.

GARRETON, Matias, PALACIOS, Pía; TRUFFELLO, Ricardo. (2016), “El Barrio como Entidad Geográfica: Revisión de Literatura y Normativas". Serie Documentos de Trabajo COES, n. 11.

GIBSON, Chris et al. (2002), "Social integration, individual perceptions of collective efficacy, and fear of crime in three cities". Justice Quarterly, v. 19, n. 3, pp. 537-564.

HALE, Chris. (1996), "Fear of crime: a review of the literature". International Review of Victimology, v. 4, n. 2, pp. 79-150.

HAN, Chunping. (2014), "Health implications of socioeconomic characteristics, subjective social status, and perceptions of inequality: an empirical study of China". Social Indicators Research, v. 119, n. 2, pp. 495-514.

HASTINGS, Annette. (2007), "Territorial justice and neighbourhood environmental services: a comparison of provision to deprived and better-off neighbourhoods in the UK". Environment and Planning C: Government and Policy, v. 25, n. 6, pp. 896-917. 
HAWDON, James et al. (2014), "Social responses to collective crime: assessing the relationship between crime-related fears and collective sentiments". European Journal of Criminology, v. 11, n. 1, pp. 39-56.

HINKLE, Joshua. (2014), "Emotional fear of crime vs. perceived safety and risk: implications for measuring. 'fear' and testing the broken windows thesis". American Journal of Criminal Justice, v. 40, n. 1, pp. 147-168.

HUMMELSHEIM, Dina et al. (2010), "Social insecurities and fear of crime: a cross-national study on the impact of Welfare State policies on crime-related anxieties". European Sociological Review, v. 27, n. 3, pp. 327-345.

HUNTER, Albert. (1978), Symbols of incivility: social disorder and fear of crime in urban neighborhoods. Paper presented to the Annual Meeting of the American Criminological Society, Dallas, november 1978.

JACKSON, Jonathan; GRAY, Emily. (2010), "Functional fear and public insecurities about crime". British Journal of Criminology.

JACKSON, Jonathan. (2004), "Experience and expression social and cultural significance in the fear of crime". British Journal of Criminology, 1 novembre 2004. v. 44, n. 6, pp. 946-966.

. (2006), Introducing fear of crime to risk research. Risk Analysis, 1 février 2006. v. 26, n. 1, pp. 253-264.

KANAN, James; PRUITT, Matthew. (2002), "Modeling fear of crime and perceived victimization risk: the (in)significance of neighborhood integration". Sociological Inquiry, v. 72, n. 4 , pp. $527-548$.

KESSLER, Gabriel. (2009), El sentimiento de inseguridad. Sociología del temor al delito. Buenos Aires, Siglo XXI.

KILLIAS, Martin; CLERICI, Christian. (2000), "Different measures of vulnerability in their relation to different dimensions of fear of crime". British Journal of Criminology, v. 40, n. 3, pp. 437-450.

KOONINGS, Kees; KRUIJT, Dirk (eds.). (1999), Societies of fear. the legacy of civil war, violence and terror in Latin America. London, Zed Books.

KROLL, Christian; DELHEY, Jan. (2013), "A happy nation? opportunities and challenges of using subjective indicators in policymaking". Social Indicators Research, v. 114, n. 1, pp. 13-28.

LAGRANGE, Randy; FERRARO, Kenneth; SUPANCIC, Michael. (1992), "Perceived risk and fear of crime: role of social and physical incivilities". Journal of Research in Crime and Delinquency, v. 29, n. 3, pp. 311-334.

LECHNER, Norbert. (1988), Los patios interiores de la democracia: subjetividad y política. México D.F., Fondo de Cultura Económica.

LINK, Felipe; VALENZUELA, Felipe; FUENTES, Luis. (2015), “Segregación, estructura y composición social del territorio metropolitano en Santiago de Chile. Complejidades metodológicas en el análisis de la diferenciación social en el espacio". Revista de Geografia Norte Grande, v. 62, pp. 151-168. 
LISKA, Allen; LAWRENCE, Joseph; SANCHIRICO, Andrew. (1982), "Fear of crime as a social fact". Social Forces, v. 60, n. 3, pp. 760-770.

LÓPEZ-MORALES, Ernesto. (2016). "Assessing exclusionary displacement through rent gap analysis in the high-rise redevelopment of Santiago, Chile". Housing Studies, v. 31, n. 5 , pp. 540-559.

LUNECKE, Alejandra. (2016), “Inseguridad ciudadana y diferenciación social en el nivel microbarrial: el caso del sector Santo Tomás, Santiago de Chile. EURE, v. 42, n. 125, pp. 109-129.

MCNEELEY, Susan; YUAN, Yue. (2017), "A multilevel examination of the code of the street's relationship with fear of crime". Crime \& Delinquency, v. 63, n. 9, pp. 1146-1167.

MÉNDEZ, María Luisa; OTERO, Gabriel. (2018). “Neighbourhood conflicts, socio-spatial inequalities, and residential stigmatisation in Santiago, Chile". Cities, v. 74, pp. 75-82.

NÚÑEZ, Javier; TOCORNAL, Ximena; HENRÍQUEZ, Pablo. (2012), “Determinantes individuales y del entorno residencial en la percepción de seguridad en barrios del Gran Santiago, Chile". Revista INVI, v. 27, n. 74, pp. 87-120.

PANTAZIS, Christina. (2000). "'Fear of crime', vulnerability and poverty". British Journal of Criminology, v. 40, n. 3, pp. 414-436.

PAYDAR, Mohammad; KAMANI-FARD, Asal; ETMINANI-GHASRODASHTI, Roya. (2017), "Perceived security of women in relation to their path choice toward sustainable neighborhood in santiago, Chile". Cities, v. 60, Part A, pp. 289-300.

PEARSON, Amber; BREETZKE, Gregory; IVORY, Vivienne. (2015), “The effect of neighborhood recorded crime on fear: does neighborhood social context matter?" American Journal of Community Psychology, v. 56, n. 1-2, pp. 170-179.

RALEIGH, Erica; GALSTER, George. (2014), "Neighborhood disinvestment, abandonment, and crime dynamics". Journal of Urban Affairs, v. 37, n. 4, pp. 367-396.

REGUILLO, Rossana. (2000), "Los laberintos del miedo. Un recorrido para fin de siglo". Revista de Estudios Sociales, v. 5, pp. 63-72.

ROLLWAGEN, Heather. (2016), "The relationship between dwelling type and fear of crime". Environment and Behavior, v. 48, n. 2, pp. 365-387.

ROTKER, Susana; GOLDMAN, Katherine. (2002), Citizens of fear: urban violence in Latin America. New Brunswick, Rutgers University Press.

RUIJSBROEK, Annemarie et al. (2015), "Social safety, self-rated general health and physical activity: changes in area crime, area safety feelings and the role of social cohesion". Health \& Place, v. 31, pp. 39-45.

SAMPSON, Robert; RAUDENBUSH, Stephen; EARLS, Felton. (1997), "Neighborhoods and violent crime: a multilevel study of collective efficacy". Science, v. 277, n. 5328, pp. 918-924.

SAMPSON, Robert; RAUDENBUSH, Stephen. (2004), "Seeing disorder: neighborhood stigma and the social construction of 'broken windows'. Social Psychology Quarterly, v. 67, n. 4 , pp. 319-342. 
SCHAFER, Joseph; HUEBNER, Beth; BYNUM, Timothy. (2006), "Fear of crime and criminal victimization: gender-based contrasts". Journal of Criminal Justice, v. 34, n. 3, pp. 285-301.

SCHIEFER, David; NOLL, Jolanda. (2017), "The essentials of social cohesion: a literature review". Social Indicators Research, DOI 10.1007/s11205-016-1314-5.

SKOGAN, Wesley. (1990), Disorder and decline: crime and the spiral of decay in american cities. New York, Free Press.

SLATER, Tom. (2013), "Your life chances affect where you live: a critique of the 'cottage industry' of neighbourhood effects research". International Journal of Urban and Regional Research, v. 37, n. 2, pp. 367-387.

VALDIVIA, Verónica; ÁLVAREZ, Rolando; DONOSO, Karen. (2012), La alcaldización de la política. Los municipios en la dictadura pinochetista. Santiago, LOM Ediciones.

VAN HAM, Maarten et al. (eds.). (2012), Neighbourhood effects research: new perspectives. Dordrecht, Springer.

VILALTA, Carlos. (2011), "Fear of crime in gated communities and apartment buildings: a comparison of housing types and a test of theories". Journal of Housing and the Built Environment, v. 26, n. 2, pp. 107-121.

WARR, Mark. (1985), "Fear of rape among urban women". Social Problems, v. 32, n. 3, pp. 238-250.

WILCOX, Pamela; QUISENBERRY, Neil; JONES, Shayne. (2003), “The built environment and community crime risk interpretation". Journal of Research in Crime and Delinquency, v. 40 , n. 3, pp. 322-345.

WILSON, James; KELLING, George. (1982), "The police and neighborhood safety: broken windows". Atlantic monthly, v. 3, pp. 29-38.

WYANT, Brian. (2008), "Multilevel impacts of perceived incivilities and perceptions of crime risk on fear of crime isolating endogenous impacts". Journal of Research in Crime and Delinquency, v. 45, n. 1, pp. 39-64.

ZHAO, Jihong; LAWTON, Brian; LONGMIRE, Dennis. (2015), “An examination of the micro-level crime-fear of crime link". Crime $\mathcal{E}$ Delinquency, v. 61, n. 1, pp. 19-44. 


\section{RESUMO}

Insegurança Percebida nos Bairros de Santiago do Chile: A Importância do Bem-Estar Subjetivo

Neste artigo estudamos os determinantes dos sentimentos de insegurança e o medo do crime em Santiago, Chile. Propomos um enfoque teórico integrador em torno ao conceito de "bem-estar", o qual permite resgatar tanto as dimensões objetivas quanto as subjetivas expostas na literatura, assim como os distintos contextos analisados: individuais e geográficos. Utilizamos dados da Pesquisa COES de Coesão e Conflito dos Bairros e dados georreferenciados no âmbito da área censitária. Os modelos de regressão linear indicam que as variáveis que capturam o bem-estar subjetivo no contexto do bairro são as mais significativas para entender as atitudes estudadas. Especificamente, as percepções de desordem física/social e as experiências de vitimização indireta mostram associações positivas; enquanto as disposições de solidariedade institucional e apego/arraigo com a comunidade mostram associações negativas com os sentimentos de insegurança e o medo ao crime dos residentes. No que diz respeito ao bem-estar objetivo, o capital econômico individual é o único fator que mantém sua significância estatística nas estimações finais. Os fatores objetivos do bairro como os níveis de criminalidade, a concentração da pobreza/riqueza, e os serviços disponíveis da vizinhança, não aparecem como preditores significativos depois de controlar o bem-estar subjetivo no contexto do bairro.

Palavras-chave: sentimento de insegurança; medo do crime; bem-estar; coesão do bairro; América Latina

\section{ABSTRACT \\ Perceived Insecurity In The Neighborhoods of Santiago (capital of Chile): The Importance Of Subjective Well-Being}

In this article, we study the determinants of feelings of insecurity and fear of crime in Santiago, Chile. We propose an integrative theoretical approach around the concept of "well-being", which allows us to rescue both the objective and subjective dimensions exposed in the literature, as well as the different contexts analyzed: individual and geographical. We used data from the COES (The Centre for Social Conflict and Cohesion Studies) Neighborhood Cohesion and Conflict Survey and georeferenced data at the census area level. Linear regression models indicate that the variables that capture subjective well-being in the neighborhood context are the most significant for understanding the attitudes studied. Specifically, perceptions of physical/social disorder and experiences of indirect victimization show positive associations; while those of institutional 
solidarity and attachment to the community show negative associations with residents' feelings of insecurity and fear of crime. Regarding objective welfare, individual economic capital is the only factor that maintains its statistical significance in the final estimates. Objective neighborhood factors, such as crime levels, poverty/wealth concentration, and available neighborhood services, do not appear as significant predictors after controlling the subjective well-being in the neighborhood context.

Keywords: insecurity feelings; fear of crime; well-being; neighborhood cohesion; Latin America

\section{RÉSUMÉ}

Insécurité Perçue Dans Les Quartiers De Santiago Du Chili: L'importance Du Bien-Être Subjectif

Dans cet article, nous étudions les déterminants du sentiment d'insécurité et de la peur du crime à Santiago du Chili. Nous proposons une approche théorique intégrative autour de la notion de "bien-être", qui nous permet de sauver à la fois les dimensions objectives et subjectives exposées dans la littérature, ainsi que les différents contextes analysés: individuel et géographique. Nous avons utilisé des données de l'Enquête COES sur la cohésion et les conflits dans les quartiers et des données géoréférencées au niveau de la zone de recensement. Les modèles de régression linéaire indiquent que les variables qui capturent le bien-être subjectif dans le contexte du quartier sont les plus importantes pour comprendre les attitudes étudiées. Plus précisément, les perceptions des troubles physiques/sociaux et les expériences de victimisation indirecte montrent des associations positives; tandis que les dispositions de solidarité institutionnelle et d'attachement/d'enracinement avec la communauté montrent des associations négatives avec le sentiment d'insécurité et de peur du crime des résidents. En ce qui concerne le bien-être objectif, le capital économique individuel est le seul facteur qui conserve sa signification statistique dans les estimations finales. Les facteurs objectifs du quartier tels que les niveaux de criminalité, la concentration de la pauvreté/de la richesse et les services de quartier disponibles n'apparaissent pas comme des prédicteurs importants après avoir contrôlé le bien-être subjectif dans le contexte du quartier.

MOTS-CLÉS: sentiment d'insécurité; peur du crime; bien-être; cohésion de quartier; Amérique Latine 


\section{RESUMEN}

Inseguridad Percibida en los Barrios de Santiago de Chile: La Importancia del Bienestar Subjetivo

En este artículo estudiamos los determinantes de los sentimientos de inseguridad y el miedo al crimen en Santiago, Chile. Proponemos un enfoque teórico integrador en torno al concepto de "bienestar", el cual permite rescatar tanto las dimensiones objetivas como subjetivas expuestas en la literatura, así como los distintos contextos explorados: individuales y geográficos. Utilizamos datos de la Encuesta COES de Cohesión y Conflicto Barrial y datos georreferenciados al nivel de zona censal. Los modelos de regresión lineal indican que las variables que capturan el bienestar subjetivo en el contexto barrial son las más significativas para entender las actitudes estudiadas. Específicamente, las percepciones de desorden físico/social y las experiencias de victimización indirecta muestran asociaciones positivas; mientras que las disposiciones de solidaridad institucional y apego/arraigo con la comunidad muestran asociaciones negativas con los sentimientos de inseguridad y el miedo al crimen de los residentes. Respecto del bienestar objetivo, el capital económico individual es el único factor que mantiene su significancia estadística en las estimaciones finales. Los factores objetivos del barrio como los niveles de criminalidad, la concentración de la pobreza/ riqueza, y los servicios disponibles del vecindario, no aparecen como predictores significativos luego de controlar por el bienestar subjetivo en el contexto barrial.

Palabras clave: sentimientos de inseguridad; miedo al crimen; bienestar; cohesión barrial; América Latina 